\title{
Acuicultura y salud pública. La expansión de la difilobotriasis en Chile y el mundo
}

\author{
Felipe C. Cabello.
}

\section{Aquaculture and public health. The emergence of diphyllobothriasis in Chile and the world}

Recent clinical and epidemiological information, an analysis of the literature, and study of the technical aspects of Chilean salmon aquaculture indicate that this activity has the ability to expand the range of diphyllobothriasis caused by the fish tapeworm Diphyllobothrium latum. Evidence for expansion of the range of the fish tapeworm includes the emergences of clinical cases in Brazil related to consumption of salmon produced in Chile. Expansion of the range of this parasite is also suggested by an increase of its geographical range in Chile, beyond its previously endemic foci in the lakes of Regions IX and X. Prevention of further dissemination of this parasitic disease rests on an improvement of sanitation and sewage disposal around the lakes of Regions IX, X and XI in Chile, improvement in aquaculture methods including curtailing the use of fish tapewormcontaminated lakes to grow juveniles forms of salmonids and more measures to decrease the number of salmonid escapees from marine pens to prevent their return to rivers and lakes carrying the infestation. Moreover, tracking the origin of juveniles in marketed salmon, determining the presence of plerocercoids in them, and increased education of the public regarding the potential dangers of eating raw fish should also be implemented. Only by stimulating the dialogue between the industry, consumers and state regulators will it be possible to implement appropriate measures to prevent further expansion of this parasitic disease by salmon aquaculture (Rev Méd Chile 2007; 135: 1064-71).

(Key words: Aquaculture; Diphyllobothriasis; Fisheries; Salmon)

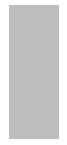

Recibido el 16 noviembre, 2006. Aceptado el 3 de enero, 2007.

Department of Microbiology and Immunology, New York Medical College, Valhalla NY, USA.

Miembro Honorario de la Academia de Medicina, Instituto de Chile. Miembro Correspondiente de la Academia de Ciencias, Instituto de Chile.

«a endemia difilobotriásica de la región de los lagos de Chile,....., representa un imperativo para la rápida solución de las precarias condiciones de saneamiento existentes en ella»

Profesor Amador Neghme, 1951

\begin{abstract}
A la memoria del profesor Amador Neghme R. parasitólogo sin par, salubrista visionario y maestro de generaciones. Un resumen de este artículo fue publicado como un comentario, "The role of salmon aquaculture in the transmission of the fish tapeworm», en Emergent Infections Diseases 2007; 13: 169-71.
\end{abstract}

Correspondencia a: Dr. Felipe Cabello. Department of

Microbiology and Immunology, New York Medical College.

Valhalla, NY 10895. Phone: 91459441 82. Fax: 9144944176.

E mail: cabello@nymc.edu 
$\mathrm{L}$ a acuicultura de salmonídeos es una industria de rápida expansión en nuestro país y en muchos otros del mundo y con un mercado cuya globalización crece diariamente ${ }^{1-4}$. La industria tiene, sin lugar a dudas, un número de beneficios económicos; pero también, ella acarrea una serie de efectos negativos para la salud humana y la animal y para el medio ambiente ${ }^{1-4}$. La acuicultura del salmón está implicada en la transmisión de enfermedades infecciosas ${ }^{1-4}$. Por ejemplo, la acuicultura de salmón en jaulas puede ser un vehículo para la transmisión de enfermedades bacterianas y parasitarias a los peces silvestres ${ }^{1,2,5,6}$, y los peces criados en estas condiciones se hacen también más susceptibles a los microorganismos y parásitos de los peces silvestres ${ }^{1,2}$. Está demostrado que la ingestión de peces silvestres puede transmitir diversos helmintos a humanos ${ }^{7}$; sin embargo, existen escasos ejemplos de la transmisión de helmintos a humanos por peces criados en acuicultura ${ }^{7}$. En este trabajo analizo y discuto información publicada y concluyo que la acuicultura del salmón en Chile está expandiendo el rango geográfico de la difilobotriasis en animales y humanos en el país y en el mundo.

\section{LOS HECHOS Y LA INVESTIGACIÓN}

Recientes publicaciones científicas provenientes de Brasil informan de la aparición de un brote de varios casos humanos de infección producidos por la tenia del pescado Diphyllobotrium latum, en varias ciudades de ese país ${ }^{8-13}$. Desde el punto de vista epidemiológico estos casos clínicos de difilobotriasis en Brasil fueron totalmente inesperados porque este parásito era totalmente desconocido para los clínicos y parasitólogos brasileños y -además-, el parásito carecía de un ciclo endémico conocido en ese país ${ }^{8-13}$. En estas publicaciones el estudio epidemiológico demostró que la fuente probable de infección en varios de estos casos de difilobotriasis fue el consumo de sushi y sashimi preparado con salmón crudo del Atlántico (Salmo salar) proveniente de Puerto Montt, en el sur de Chile ${ }^{9,10}$. Las infecciones por D. latum fueron diagnosticadas en varias ciudades de Brasil, incluyendo Río de Janeiro, Sao Paulo y Salvador ${ }^{8-13}$, e incluso en un turista europeo que viajara a este país, sugiriendo una amplia disemi- nación de la fuente infecciosa ${ }^{13}$. La aparición de casos de difilobotriasis en Brasil ha llevado a las autoridades sanitarias locales a estudiar la instauración de medidas higiénicas para prevenir el aumento de infecciones humanas con esta parasitosis ${ }^{8-12}$.

La infección humana y animal por D. latum se adquiere por la ingestión de plerocercoides (larvas) de este parásito presentes en la carne y vísceras de pescado infectado y que es consumido crudo o con insuficiente cocción (Figura 1) ${ }^{14}$. D. latum, y el cestodo del mismo grupo la tenia de la gaviota, D. dendriticum, tienen ciclos endémicos bien establecidos en las aguas y las riveras de la serie de lagos y lagunas glaciares de las regiones IX y X del sur de Chile, incluyendo los lagos Colico, Panguipulli, Rupanco y Llanquihue ${ }^{15-24}$. Infecciones humanas y animales con estos parásitos han sido detectadas en esta región chilena desde los años 50, como resultado de la ingestión de pescado sin cocinar, generalmente ahumado, proveniente de estos lagos ${ }^{15-24}$. El eslabón que cierra la cadena epidemiológica entre la epidemia brasileña de difilobotriasis y la acuicultura del salmón en los lagos glaciares del sur de Chile, donde existe una endemia de D. latum y D. dendriticum, se produce al ser -algunos de estos lagos-, usados por la industria de acuicultura del salmón para crecer en jaulas las formas juveniles de estas especies ${ }^{25-27}$. El desarrollo en estos lagos como el Ranco, el Puyehue, el Rupanco, el Llanquihue y el Yelcho ${ }^{25}$ de las formas juveniles de salmonídeos es usada por la industria chilena de acuicultura para acelerar el crecimiento de ellas, ya que en ellos este crecimiento es más rápido y menos costoso que en piscinas artificiales terrestres construidas para este objeto, las que deben usar agua filtrada y aun esterilizada con luz ultravioleta 26,27 . En estas jaulas lacustres las formas juveniles de salmonídeos están expuestos a las aguas sin filtrar de estos lagos e ingieren copépodos presentes en el zooplancton, y a lo mejor también pequeños peces, conteniendo las formas larvales del difilobotrio (Figura 1) ${ }^{14,21}$. Subsecuentemente, estas formas salmonídeas juveniles infectadas con larvas de difilobotrio y maduras para tolerar la presión osmótica del mar son transportadas a éste, donde en jaulas alcanzan el tamaño adulto necesario para su comercialización ${ }^{25-27}$. La práctica de crecimiento de las formas 


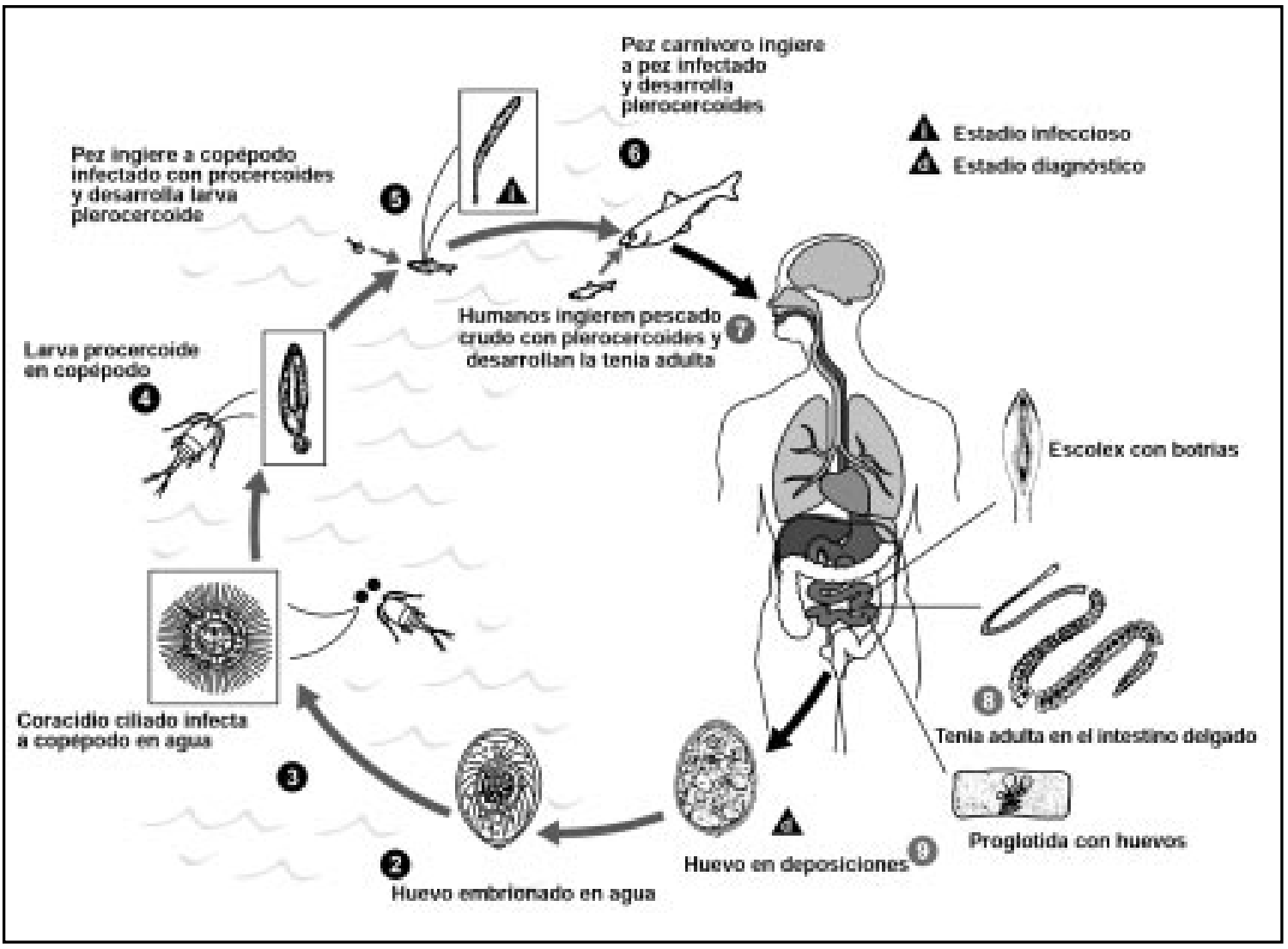

Figura 1. Ciclo biológico del Difilobotrium latum tomado y adaptado de http://www.dpd.cdc.gov/dpdx

juveniles de salmonídeos en jaulas en los lagos es -al parecer-, un método único de la industria de acuicultura chilena, ya que ella no está permitida, y aun es evitada por razones sanitarias, por la industria acuícola de otros países como Noruega, Irlanda, Escocia, EE.UU. de Norteamérica y Canadá 26,27 .

En los últimos 55 años el trabajo de una serie de parasitólogos chilenos, comenzando por el maestro de la parasitología chilena A. Neghme y continuando con P. Torres y su grupo, ha demostrado que carne de especies nativas y de especies salmonídeas introducidas a los lagos de las regiones IX y X ya descritos, están densamente infectadas con plerocercoides de Diphyllobothrium ${ }^{15-24}$. Los mismos trabajos han demostrado que los huéspedes intermediarios de la tenia del pescado, los copépodos calanoides Diaptomus diabolicus y
Boeckela gracilipes están representados de manera abundante en el zooplancton en el agua de estos lagos (Figura 1) ${ }^{21}$. De esta forma los peces nativos y las especies salmonídeas introducidas a estos lagos ingieren los copépodos conteniendo las larvas procercoides de Dyphillobotrium, las cuales se desarrollan en plerocercoides, en la carne de estos peces lo que la hace infecciosa para humanos y animales (Figura 1) ${ }^{14,21}$. Así el ciclo biológico de esta tenia es cerrado en estos lagos por la ingestión de la carne de pescado no cocinada por humanos y animales que son los huéspedes definitivos de ella (Figura 1) ${ }^{14,15-24}$. La persistencia de la endemia difilobotriásica en estos lagos es asegurada por la descarga en ellos de aguas servidas humanas sin tratamiento y de las deposiciones de animales infectados, las cuales liberan millones de huevos de este parásito, que al 
poco tiempo producen los coracidios ciliados que infectarán a los copépodos descritos anteriormente (Figura 1) 14,15-24.

Los estudios epidemiológicos brasileños citados no detectaron plerocercoides de Diphyllobothrium en varias muestras de salmón producido en Chile analizados durante y después de la presentación de los casos en Brasil ${ }^{9}$. Sin embargo, la ausencia de plerocercoides se debería a un muestreo insuficiente y a una variabilidad temporal y espacial en la infestación de los salmones con plerocercoides ${ }^{15-24}$. Esto último como resultado de que no todas las formas juveniles de salmonídeos crecen en los mencionados lagos, y si se desarrollan en ellos no todos los juveniles ingieren las mismas cantidades de copépodos infectados con procercoides de Diphyllobothrium $^{15-24}$. Además, los diferentes lagos pueden tener diversas densidades de copépodos y éstos pueden estar infectados con frecuencias diferentes a lo largo del tiempo ${ }^{15-21}$. Sin embargo, trabajos en Chile han demostrado una alta infestación en la carne de peces de especies nativas e introducidas en estos lagos ${ }^{18}$, y la presencia de plerocercoides de Diphyllobothrium en la trucha arco iris (Onchorhyncus mykiss), criada en recintos de acuicultura, hace sugerir que animales criados en estas condiciones tienen la posibilidad de infectarse y desarrollar la presencia de plerocercoids de Diphyllobothrium en su carne ${ }^{28}$. En Chile, la infestación con plerocercoides de Diphyllobothrium ha sido también detectada en el salmón coho (Oncorhynchus kisutch) ${ }^{29}$, una especie de salmonídeos introducida al país y que vive en el ambiente silvestre como resultado de haber escapado de jaulas mantenidas en el mar, en el proceso de su crianza industrial ${ }^{3,25,30}$. Larvas plerocercoides de otra tenia del pescado, D. pacificum, cuyo huésped definitivo son mamíferos marinos como las focas y los lobos marinos, también han sido detectados en la carne de otros peces chilenos y han producido algunos casos de difilobotriasis en el norte del país ${ }^{31,32}$. Es bien sabido que las jaulas de acuicultura en el mar atraen a estos mamíferos y esto crearía la posibilidad de instaurar el ciclo biológico de este difilobotrio alternativo alrededor de estas jaulas ${ }^{31,32}$. Pero este escenario parece ser poco probable para explicar las infestaciones por Diphyllobothrium en Brasil ya que los parásitos diagnosticados en ese país tenían las característi- cas morfológicas de D. latum, que es el parásito humano endémico en los lagos de las regiones IX y $\mathrm{X}^{9,15-24}$ y la presencia de D. pacificum no ha sido aún descrita en los mares del sur de Chile. Similarmente parece poco probable que los casos de teniasis en Brasil hayan sido producidos por D. dendriticum, ya que los huéspedes definitivos de esta tenia son algunas gaviotas y la tenia adulta muy raramente se ha encontrado infectando humanos 33,34 .

Este análisis sugiere, claramente, que la acuicultura del salmón en Chile ha expandido el rango de huésped de la difilobotriasis a especies de salmonídeos introducidas en el país por esta industria como son el salmón coho (O. kisutch) y el salmón del Atlántico (S. salar) ${ }^{8-13,28}$. Esto ha sido posible por la crianza de juveniles de estas especies en lagos donde las formas larvales de la difilobotriasis existen en los huéspedes intermediarios de ésta de manera endémica ${ }^{15-24,28,29}$. El análisis también sugiere que, probablemente, la acuicultura del salmón está expandiendo además el rango geográfico de la difilobotriasis en Chile, a través de por lo menos dos mecanismos. El primero, producido por el escape de salmones infestados con plerocercoides de difilobotrio de las jaulas marinas ${ }^{28-30}$. El retorno de estos peces con plerocercoides a ríos como el Simpson en la XI región abre la posibilidad para la expansión de la endemia, ya que la ingesta de carne sin cocinar de estos salmones por humanos y por animales en la cuenca del río Simpson, producirá en ellos las formas adultas del parásito y con ello el pasaje de huevos en las deposiciones que contaminarán lagos y lagunas en la cuenca de este torrente, expandiéndose potencialmente con ello el ciclo de la difilobotriasis en una nueva región del país ${ }^{29,30}$. Similarmente, la ingesta de carne de salmón sin cocinar conteniendo plerocercoides por habitantes de otras regiones del país, también tiene el potencial de expandir el rango de la parasitosis a ellas, si es que las aguas servidas que contienen las deposiciones de individuos infectados con la forma adulta del parásito alcanzan cuerpos de agua que contienen las especies necesarias para mantener el ciclo vital del parásito ${ }^{15-24}$. El análisis también indica que la globalización del mercado de salmón, cultivado en el sur de Chile, tiene el potencial de expandir el rango de la parasitosis a otros países como se ha 
demostrado en el Brasil ${ }^{8-13}$. Especialmente, si en esos países existen las condiciones para la instauración de nuevos nichos de la parasitosis i. e. falta de tratamiento de aguas servidas depositadas en cuerpos de agua dulce conteniendo las especies de copépodos y de peces necesaria para la propagación del difilobotrio.

Los fenómenos descritos en los párrafos precedentes tienen sus antecedentes en la acuicultura del salmón en Escocia y en Noruega, donde a comienzos de la instalación de estas industrias en dichos países 30 años atrás se describieron severas infestaciones con plerocercoides de difilobotrium de salmón y trucha, que fueron acompañadas de gran mortalidad de los salmonídeos juveniles criados en jaulas en lagos de agua dulce e, $6,35,36^{\text {. La }}$ presencia de este fenómeno, y de otro tipo de infestaciones como la producida por el alga Saprolegna, un fenómeno que también ocurre en los lagos del sur de Chile, obligó a las industrias acuícolas de aquellos países a descartar el uso de lagos de agua dulce en la crianza industrial de salmonídeos a pesar de ser este método económicamente más rentable que la construcción de piscinas terrestres ${ }^{25,35,36}$. Ocasionalmente también, se han descrito casos de difilobotriasis asociadas a la ingesta de salmón crudo silvestre, probablemente, como resultado de que las formas juveniles de ellos han crecido en lagos con endemia para difilobotriasis, como ha sucedido en Alaska $^{37,38}$.

\section{CONCLUSIONES}

El análisis realizado indica que el fenómeno epidemiológico descrito es un ejemplo más de los peligros sanitarios que encierra la globalización de la producción alimenticia como resultado de la industrialización de la crianza de animales ${ }^{1-4}$ y de la globalización comercial y económica ${ }^{1-4}$. La globalización de la industria alimenticia genera, simultáneamente, cambios en los hábitos alimenticios como la ingesta de carne de pescado crudo en sushi y en ceviche que hacen también posible la diseminación global de patógenos como el difilobotrio ${ }^{1-4,7}$. La expansión del rango geográfico de la endemia por difilobotrio en Chile, por la acuicultura, por los mecanismos descritos en esta discusión tiene el potencial de aumentar los casos humanos de ésta en el país y en el extranjero si ella no es controlada efectivamente. Esto afectará, potencialmente, la salud económica de la industria acuicola y de las regiones del país donde ésta se desarrolla ya que la comercialización de un producto que pueda estar infestado con difilobotrium puede ser restringida por organismos sanitarios ${ }^{1,4,10}$. Es indudable también, que en Chile la salmonicultura está actuando como un amplificador de esta endemia ya que al aumentar y concentrar, masivamente, la población de peces susceptibles en los lagos conteniendo los intermediarios de difilobotrio aumenta la carga de infección y lo mismo sucede con los cientos de miles de peces escapados de las jaulas marinas, potencialmente infectados, y con su posible regreso a ríos y lagos de otras regiones del país creando nuevos focos de infección ${ }^{28-30}$. Este problema es exacerbado también porque la industria acuicola chilena no respeta los convenios de producción, produciendo en exceso las cantidades fijadas por los organismos gubernamentales que regulan la industria ${ }^{39}$. Como ha sido el caso con otras enfermedades infecciosas diseminadas por la industrialización de la crianza de animales, como la de las vacas locas y la influenza aviar ${ }^{40}$; esta expansión del nicho de la difilobotriasis podría haberse evitado usando la información producida en Chile durante los últimos 50 años respecto de la endemia de este parásito en los lagos del sur de Chile ${ }^{15-24}$ y la capacidad de esta parasitosis para ser trasmitida por la ingesta de pescado crudo ${ }^{15-24}$.

Tradicionalmente, en Chile las infecciones por difilobotriasis se diagnosticaban en individuos que habían ingerido pescado ahumado y sin cocinar proveniente de los lagos de las regiones IX y $\mathrm{X}^{18-}$ 23. La infección también es frecuente en los animales domésticos y silvestres viviendo en las riveras de estos lagos ${ }^{15-24}$. En Europa y Norteamérica, las infecciones con la tenia del pescado eran frecuentes en mujeres de ascendencia judía, las cuales en la preparación del plato tradicional de esta cultura «gefilte fish», que es preparado con peces de carne blanca pescados en lagos, ingerían trocitos de carne de estos peces sin cocinar infectados con plerocercoides de difilobotrio ${ }^{40-43}$. Como este análisis lo demuestra es posible también infectarse con este parásito con la ingestión de carne infestada con plerocercoides sin cocinar en sushi y sashimi, y probablemente también, en ceviches $^{8-13}$. Esta enfermedad puede ser preveni- 
da cocinando el pescado a $54^{\circ} \mathrm{C}$ o $56^{\circ} \mathrm{C}$ por 5 $\min ^{44}$. Alternativamente, los plerocercoides pueden ser destruidos por congelamiento rápido de la carne de pescado a $-35^{\circ} \mathrm{C}$ por $15 \mathrm{~h}$ o por congelamiento habitual a $-20^{\circ} \mathrm{C}$ por 7 días antes del consumo ${ }^{42}$. Está claro que para evitar nuevos brotes de esta enfermedad en Chile y en el extranjero y para limitar y disminuir la endemia de la difilobotriasis en el país, el salmón criado en acuicultura no debiera comerse crudo a menos que pueda certificarse que las formas juveniles no han sido cobijadas en los lagos en que la difilobotriasis es endémica. A largo plazo es obvio que la industria acuicola chilena tiene que disminuir y finalmente eliminar totalmente la tecnología que requiere del uso de los lagos el sur de Chile para hacer crecer las formas juveniles de salmoni$\operatorname{deos}^{24,25}$, y en este proceso ponerse a la par con los aspectos sanitarios de las industrias salmoneras del hemisferio norte ${ }^{24,25}$. Las instituciones del Estado encargadas de velar por la salud humana y animal debieran también establecer e implementar las regulaciones necesarias para mejorar el saneamiento ambiental (propuesto por el Profesor Neghme 56 años atrás), de los lagos de las

\section{REFERENCIAS}

1. Naylor $R$, Burke M. Aquaculture and ocean resources: Raising tigers of the sea. Annu Rev Environ Resour 2005; 30: 185-218.

2. Goldburg RJ, Eшiot MS, Naylor RL Marine aquaculture in the Unites States. Arlington, VA: Pew Ocean Commission, 2001.

3. Bushman H, Riquelme VA, Hernández-Gonzales MC, Vareia D, JimÉnez JE, Henríquez LA et al. A review of the impacts of salmonid farming on marine coastal systems in the southeast Pacific. ICES J Marine Sci 2006; 63: 1338-45.

4. Jensen GL, Greenlees KJ. Public health issues in aquaculture. Rev Sci Tech Off Int Epiz 1997; 16: 641-51.

5. KRKoseK M, Lewis MA, Volpe JP. Transmission dynamics of parasitic sea lice from farm to wild salmon. Proc R Soc B 2005; 272: 689-96.

6. Krkosek M, Lewis MA, Morton A, Frazer LN, Volpe JL. Epizootics of wild fish induced by farm fish. Proc Natl Acad Sci USA 2006; 103: 15506-10. regiones IX, X y XI con endemia de la tenia del pescado, y para regular que la industria acuicola mantenga las condiciones sanitarias que eviten la propagación de ésta y otras enfermedades infecciosas. Además, debiera controlarse la calidad de los productos de acuicultura comercializados y consumidos en el país y los exportados al extranjero, y educar a la población respecto de los problemas potenciales de la ingesta de pescado crudo, ya sea ahumado o en sushi, sashimi y ceviche.

\section{Agradecimientos}

El autor hizo esta investigación bajo los auspicios de una beca de la fundación John Simon Guggenheim. Agradezco a mis colegas P. Torres, M. Georges-Nascimento, R. Neira, A. Buschman, G. Fursi y A. Fortt de Chile, J.L.M. Sampaio de Brazil, y L Aarflot y A. Lyngoy de Noruega por información. Betty Barría y H. Harrison me dieron una ayuda invaluable en la preparación del manuscrito. J. Gallardo, S. Leyton y J. Hernández de la Biblioteca de la Escuela de Medicina, Universidad de Chile, me ayudaron de manera importante con la bibliografía chilena.

7. Chai J-Y, MurRel KD, Lymbery AJ. Fish-borne parasitic zoonoses: Status and issues. IntJ Parasitol 2005; 35: 1233-54.

8. Paula Eduardo MB, Sampaio JLM, Nascimento Gonçalves EM, Castilho VLP, Randi AP, Thiago C et al. Diphyllobothrium spp.: um parasita emergente em Sao Paulo, Associado ao Consumo de Peixe Cru - Sushis e Sashimis, Sao Paulo, Marco de 2005. Bol Epidemiol Paulista 2005; 2 (15).

9. Eduardo MBP, Sampaio JLM, Cesar MLVS, Gonçalves EMN, Castilho VLP, Albuquerque SMSR et al. Investigacao epidemiologica do surto de difilobotriase, Sao Paulo, maio de 2005. Bol Epidemiol Paulista 2005; 2 (17).

10. Sampaio JlM, Andrade VP, Lucas MC, Fung L, Gagliardi SMB, Santos SRP et al. Diphyllobothriasis, Brazil. Emerg Infect Dis 2005; 11: 1598-600.

11. Santos FLN, Faro LB. The first confirmed case of Diphyllobothrium latum in Brazil. Mem Inst Oswaldo Cruz, Rio de Janeiro 2005; 100: 685-6. 
12. Tavares LER, Luque JL, Do Bomfim TCB. Human diphyllobothriasis: Reports from Rio de Janeiro, Brazil. Rev Brax Parasitol Vet 2005; 14: 85-7.

13. Van Doorn HR, Van Vugt M, WetSTEYN JC, Van GooL T. Infestation with the tapeworm Diphyllobothrium latum after eating raw fish. Ned Tijdschr Geneeskd 2005; 149: 2470-2.

14. Dick TA, Nelson PA, Choudhury A. Diphyllobothriasis: Update on human cases, foci, patterns and sources of human infections and future considerations. Southeast Asian J Trop Med Public Health 2001; 32 (Suppl 2): 59-76.

15. Neghme A, Donckaster R, Silva R. Diphyllobothrium latum en Chile. Primer caso autóctono en el hombre. Rev Méd Chile 1950; 78: 410-1.

16. Neghme A, Bertín V, Tagie I, Silva R, Artigas J. Diphyllobothrium latum en Chile. II. Primera encuesta en el Lago Colico. Bol Inform Parasitol Chile 1950; 5: 16-7.

17. Neghme DA, Bertin V. Diphyllobothrium latum in Chile. IV. Estado actual de las investigaciones epidemiologicas. Rev Chil Hig Med Prev 1951; 13: 8-11.

18. Torres P, Cubilos V, Gesche W, Reboledo C, Montefusco A, Miranda C et al. Difilobotriasis en salmonidos introducidos en lagos del sur de Chile: Aspectos patológicos, relación con infección humana, animales domésticos y aves piscívoras. Arch Med Vet 1991; 23: 165-83.

19. Torres P, Franjola R, Weitz JC, Pena G, Morales E. Registro de nuevos casos de diffilobotriasis humana en Chile (1981-1992), incluido un caso de infección múltiple por Diphyllobothrium latum. Bol Chil Parasitol 1993; 48: 39-43.

20. Torres P, Franjola R, Figueroa L, Schiatter R, González H, Contreras B et al. Researches on Pseudophyllidea (Carus, 1813) in the south of Chile. IV. Occurrence of Diphyllobothrium dendriticum (Nitzch). J Helminthol 1981; 55: 173-87.

21. Torres P, Viшalobos L, Woelfl S, Puga S. Identification of the copepod intermediate host of the introduced broad fish tapeworm Diphyllobothrium latum, in Southern Chile. J Parasitol 2004; 90: 1190-93.

22. Torres P, Cuevas C, Tang M, Barra M, Franjota $R$, NAVARRETE $\mathrm{N}$ ET AL. Introduced and native fishes as infection foci of Diphyllobothrium spp. in humans and dogs from two localities at Lake Panguipulli in Southern Chile. Comp Parasitol 2004; 71: 111-7.
23. Kurte C, Silva M, Gajardo E, Torres P. Nuevos casos de difilobotriasis humana en Panguipulli, Chile. Bol Chil Parasitol 1990; 45: 59-61.

24. Torres P, Gesche W, Montefusco A, Miranda JC, Dietz P, Huijse R. Diphyllobothriosis humana y en peces del lago Riñihue, Chile: efecto de la actividad educativa, distribución estacional y relación con sexo, talla y dieta de los peces. Arch Med Vet 1998; 30: 31-45.

25. Soto M, Arismendi I, Gonzales J, Sanzana J, Jara F, JaRA C ET al. Sur de Chile, país de truchas y salmones: patrones de invasión y amenazas para las especies nativas. Rev Chilena Hist Nat 2006; 79: 97-117.

26. Beveridge M. Cage Aquaculture. $3^{\text {rd }}$ edition. UK: Blackwell Publishing Ltd, 2004.

27. SteAd SM, LaIRD L Handbook of Salmon Farming. Chichester, UK: Praxis Publishing Ltd, 2002.

28. Torres P, López JC, Cubilos V, Lobos C, Silva R. Visceral diphyllobothriosis in a cultured rainbow trout, Oncorhynchus mykiss (Walbaum), in Chile. J Fish Dis 2002; 25: 375-9.

29. Torres P, Aedo E, Figueroa L, Siegmund I, Silva R, NAVARRETE N ET AL. Infección por helmintos parásitos en salmón coho, Oncorhynchus kisutch, durante su retorno al río Simpson, Chile. Bol Chil Parasitol 2000; 55: 31-5.

30. Soto D, JaRA F, Moreno C. Escaped salmon in the inner seas, southern Chile: Facing ecological and social conflicts. Ecological Applications 2001; 11: 1750-62.

31. Sagua FH, Aluaga FP, Neira CI, Araya RJ, González C. Diphyllobothriosis humana por infección por Diphyllobothrium pacificum en un niño de 3 año en Antofagasta, Chile. Rev Chil Pediatr 2000; 71: 427-9.

32. Sagua H, Neira I, Araya J, González J. Nuevos casos de infección humana por Diphyllobothrium pacificum (Nybelin, 1931) Margolis, 1956 en Chile y su probable relación con el fenómeno de El Niño, 1975-2000. Bol Chil Parasitol 2001; 56: 22-5.

33. González H, Garrido V, Martens P, Aguirrebeña R. Identificación de Diphyllobothrium sp. en especies salmonídeas del Lago Rupanco, Chile. Bol Chile Parasitol 1978; 33: 25-34.

34. González H, Garrido V, Landeta ME, Martens P. Nuevos aportes para la identificación de Diphyllobothrium sp., en el Lago Rupanco, Chile. Bol Chile Parasitol 1980; 35: 10-4. 
35. Wooten R, SmTth JW. The occurrence of plemocercoids of Diphyllobothrium spp. in wild and cultured salmonids from the Locch Awe area. Scottish Fisheries Research Report number 13. 1979. ISNN 03088022.

36. MATHESON AA. An investigation of the occurrence and control of plerocercoids of Diphyllobothrium spp. In farmed Atlantic salmon (Salmo salar). 1980. M Sci Thesis University of Stirling. U. Kingdom.

37. Turner JA, SorviLo FJ, Murray RA, Chin J, MidDAUgh JP, Dietrich PD ET aL Diphylobothriasis associated with salmon - United States. MMWR 1981; 30: 331-8.

38. Hutchinson JW, Bass JW, Demers DM, Myers GB. Diphyllobothriasis after eating raw salmon. Hawaii Med J 1997; 56: 176-7.

39 Sobreproducción salmonera: El fracaso de los acuerdos voluntarios. Publicaciones Terram, 2006. Mayo. Número 9. http://www.terram.cl/ docs/salmon9_mayo06.pdf
40. GiBBs EPJ. Emerging zoonotic epidemics in the interconnected global community. The Veterinary Record 2005; 157: 673-9.

41. Nawa Y, Hatz C, Bum J. Sushi delights and parasites: The risk of fishbome and foodborne parasitic zoonoses in Asia. Clin Infect Dis 2005; 41: 1297-303.

42. Butt AA, Aidridge KE, Sanders CV. Infections related to the ingestion of seafood. Part II: parasitic infections and food safety. The Lancet Infect Dis 2004; 4: 294-300.

43 Peduzzi R, Boucher-Rodoni R. Resurgence of human bothriocephalosis (Disphyllobothrium latum) in the subalpine lake region. J Limnol 2001; 60: 41-4.

44. US Food and Drug Administration (2001) Processing parameters needed to control pathogens in cold smoked fish. Potential hazards in coldsmoked fish: Parasites Center for Food Safety and Applied Nutrition. March 29, 2001. http:// www.cfsan.fda.gov/ comm/ift2para.htm1 